\title{
Ferroelectric and octahedral tilt twin disorder and the lead-free piezoelectric, sodium potassium niobate system
}

\author{
Jason Schiemer, Ray L. Withers*, Yun Liu, Zhiguo Yi \\ Research School of Chemistry, The Australian National University, Canberra, ACT 0200, Australia
}

\section{A R T I C L E I N F O}

Available online 10 February 2012

Keywords:

Structured diffuse scattering

Order and disorder

Ferroelectric

Lead-free

Electron diffraction

\begin{abstract}
A B S T R A C T
Using electron diffraction, trends in the local structural behaviour of the $\mathrm{K}_{x} \mathrm{Na}_{1-{ }_{-}} \mathrm{NbO}_{3}$ (KNN $x$ ) 'solid solution' system are investigated and interpreted using an order/disorder based theoretical framework. At room temperature, electron diffraction shows a single plane of transverse polarised, diffuse intensity perpendicular to $\left[\begin{array}{lll}0 & 1 & 0\end{array}\right]_{p}^{*}$ ( $p$ for parent sub-structure) across the entire phase diagram, indicative of ferroelectric disorder along the $\left[\begin{array}{lll}0 & 1 & 0\end{array}\right]_{p}$ direction co-existing with long range ferroelectric order along the orthogonal $\left[\begin{array}{lll}1 & 0 & 0\end{array}\right]_{p}$ and $\left[\begin{array}{lll}0 & 0 & 1\end{array}\right]_{p}$ directions. An additional characteristic pattern of diffuse scattering is also observed, involving rods of diffuse intensity running along the $\left[\begin{array}{lll}1 & 0 & 0\end{array}\right]_{p}^{*}$ and $\left[\begin{array}{lll}0 & 0 & 1\end{array}\right]_{p}{ }^{*}$ directions of the perovskite sub-structure and indicative of octahedral tilt disorder about the $\left[\begin{array}{llll}1 & 0 & 0\end{array}\right]_{p}$ and $\left[\begin{array}{lll}0 & 0 & 1\end{array}\right]_{p}$ axes co-existing with long range ordered octahedral tilting around the $\left[\begin{array}{lll}0 & 1 & 0\end{array}\right]_{p}$ direction. A possible crystal chemical explanation for the existence of this latter octahedral tilt disorder is explored through bond valence sum calculations. The possible influence of both types of disorder on the previously refined, room temperature space group/s and average crystal structure/s is examined.
\end{abstract}

(c) 2012 Elsevier Inc. All rights reserved.

\section{Introduction}

After the publication of strong, room temperature, piezoelectric properties by Saito et al. in 2004 [1], the $\mathrm{K}_{x} \mathrm{Na}_{1-x} \mathrm{NbO}_{3}$ (KNN $x$ ) solid solution and doped variants thereof recently re-emerged as (one of) the most promising lead-free piezoelectric ceramic systems and has remained the leading candidate since. Relatively high piezoelectric coefficients (up to $\sim 300 \mathrm{pC} / \mathrm{N}$ ) along with Curie temperatures of up to $400{ }^{\circ} \mathrm{C}$ make it one of only a few systems able to compete with the current most widely used, lead oxide based systems. So-called "morphotropic phase boundaries" have been reported at $x \sim 0.5,0.35$ and 0.2 . Further structural investigations have discounted any significant structural change at the $x=0.35$ composition, although inconsistent reports of discrepancies in properties around this composition exist [2,3].

Significantly enhanced piezoelectric properties, however, do occur in the KNN $x$ system when the polymorphic phase transition boundary from the 'orthorhombic' ferroelectric state (usually reported with an apparent small monoclinic distortion) to the tetragonal ferroelectric state (the O-T transition) is shifted down to room temperature through doping, as was achieved in Saito et al.'s [1] well-known work. The enhanced piezoelectric response is then highest at the polymorphic phase transition boundary,

\footnotetext{
* Corresponding author. Fax: +61261250750.

E-mail address: withers@rsc.anu.edu.au (R.L. Withers).
}

although the excellent piezoelectric properties of the region have poor stability with regard to changing temperature [4]. Using doped compositions with the $\mathrm{O}-\mathrm{T}$ transition below room temperature enhances the temperature stability of the properties, though at some cost to the overall magnitude of the measured piezoelectric coefficients.

The structures, both local as well as average, of materials such as KNN $x$ are thus of considerable interest, as materials scientists seek to establish structure-property relationships and to engineer improvements in properties through strategies such as doping and domain engineering. In this paper, clear evidence is presented for the existence of 1-D polar as well as octahedral tilt disorder co-existing with long range ferroelectric and octahedral tilt ordering across the entire KNN $x$ system. This co-existence of order and disorder (both ferroelectric and octahedral tilt) means that the average structure/s determined by conventional crystallographic means (see e.g., [5]) are not the same as the true local structures (see also e.g., [6-8]). In turn, this means that the local crystal chemistry underlying the ferroelectric and piezoelectric properties of perovskite-related ferroelectric materials such as KNN $x$ can never be properly understood until both the long range and short range ordering are simultaneously taken into account. This requires an order/disorder based way of thinking about the structures of such materials, as originally proposed by Comes and colleagues [6-8].

The purpose of the current paper therefore is to present the latter order/disorder based theoretical background prior to using 
it to understand and interpret the results of a detailed electron diffraction study of the co-existing long and short range ordering characteristic of the KNN $x$ system.

\section{An order/disorder based approach to the KNN $x$ system}

There are two largely independent, triply degenerate, degrees of freedom that determine the phase transition and associated structural behaviour of the KNN $x$ system. The first such triply degenerate degree of freedom is the 1-D ferroelectric, correlated off-centre displacements of $\mathrm{Nb}^{5+}\left(\right.$ and $\left.\mathrm{Na}^{+} / \mathrm{K}^{+}\right)$ions relative to the oxygen ion octahedral framework along all three $\left\langle\begin{array}{llll}0 & 0 & 1\end{array}\right\rangle_{p}$ (subscript $p$ for parent perovskite sub-structure) directions, see, e.g., an [ $\left.\begin{array}{lll}0 & 0 & 1\end{array}\right]$ column of the tetragonal $P 4 \mathrm{~mm}$ average structure of $\mathrm{KNbO}_{3}$ [5] shown in Fig. 1(a). (Note that the various off-centre displacements involved have each been increased by a factor of 3 relative to these refined in [5] to make them more apparent in Fig. 1(a)). This degree of freedom is responsible for macroscopic polarisation (e.g., along the vertical [ $\left[\begin{array}{lll}0 & 0 & 1\end{array}\right]$ direction in the tetragonal $\mathrm{P4mm}$ phase of $\mathrm{KNbO}_{3}$ [5], see Fig. 1(a)) when long range transverse correlated and for 1-D nano-scale polar regions (NPR's; see, e.g., Fig. 1(d)) and corresponding $\mathbf{G} \pm\left\{\begin{array}{lll}0 & 0 & 1\end{array}\right\}^{*}$ sheets of diffuse intensity (G a parent perovskite sub-structure reflection; see a

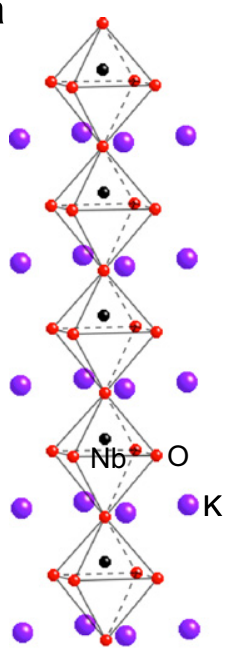

C

$\mathbf{G} \pm(001)^{\star}$ sheets of diffuse in reciprocal space

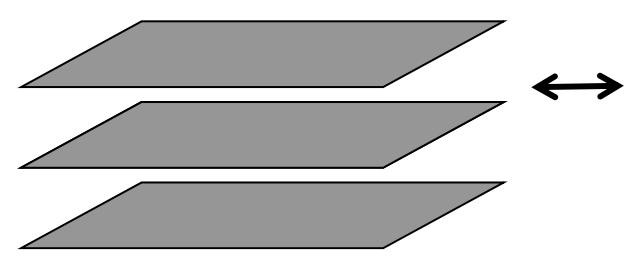

b

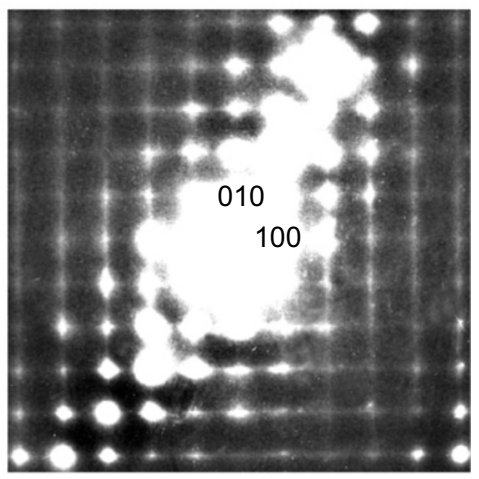

d



Fig. 1. (a) An [ $\left[\begin{array}{lll}0 & 0 & 1\end{array}\right]$ column of the tetragonal $\mathrm{P} 4 \mathrm{~mm}$ average structure of $\mathrm{KNbO}_{3}$ (note that the various off-centre displacements involved have each been increased by a factor of three relative to these refined in [5] to make them more apparent), (b) a close to [ $\left.\begin{array}{lll}0 & 0 & 1\end{array}\right]$ zone axis diffraction pattern from the tetragonal phase of $\mathrm{BaTiO}_{3}$, (c) $\mathrm{G}+\left\{\begin{array}{llll}0 & 0 & 1\end{array}\right\}^{*}$ sheets of diffuse intensity (G a parent perovskite sub-structure reflection) and (d) the equivalent real-space 1-D polar nano regions.

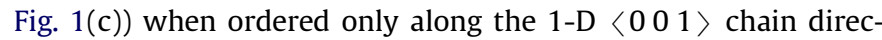
tions but not along the orthogonal, or transverse, directions [6-8].

Many years ago, Comes et al. [6] showed that the high temperature cubic phase of the end-member composition of the KNN $x$ system, $\mathrm{KNbO}_{3}$, was characterised by three separate $\mathbf{G} \pm\left\{\begin{array}{lll}0 & 0 & 1\end{array}\right\}^{*}$ sheets of diffuse intensity i.e., by 1-D NPR's along all three $\left\langle\begin{array}{lll}0 & 0 & 1\end{array}\right\rangle$ real space directions. These 1-D NPR's are strongly correlated along one particular $\left\langle\begin{array}{lll}0 & 0 & 1\end{array}\right\rangle_{p}$ direction but are not correlated from one such NPR to the next in the respective transverse directions. Consequently, “ ... even in the (paraelectric) cubic phase every unit cell is polarised at almost every instant of time: only the time (and space)-averaged macroscopic polarisation is actually zero ...." [9].

On cooling through the cubic to tetragonal phase transition, one of these sheets of diffuse disappears, corresponding to the onset of transverse correlation of the pre-existing $\left[\begin{array}{lll}0 & 0 & 1\end{array}\right]_{p}$ NPR's and to long range order and macoscopic polarisation along the [ $\left.\begin{array}{lll}0 & 0 & 1\end{array}\right]$ direction [6-8]. The other two sets of pre-existing [ $\left[\begin{array}{lll}1 & 0 & 0\end{array}\right]_{p}$ and $\left[\begin{array}{lll}0 & 1 & 0\end{array}\right]_{p}$ NPR's, however, stay correlated only along [ $\left[\begin{array}{lll}1 & 0 & 0\end{array}\right]_{p}$ and $\left[\begin{array}{lll}0 & 1 & 0\end{array}\right]_{p}$, respectively, and hence the $\mathbf{G} \pm\left(\begin{array}{lll}1 & 0 & 0\end{array}\right)^{*}$ and $\mathbf{G} \pm\left(\begin{array}{lll}0 & 1 & 0\end{array}\right)^{*}$ sheets of diffuse intensity necessarily remain in the tetragonal phase of $\mathrm{KNbO}_{3}$. The close to [ $\left[\begin{array}{lll}0 & 0 & 1\end{array}\right]$ zone axis electron diffraction pattern (EDP) of the room temperature, tetragonal $\mathrm{P} 4 \mathrm{~mm}$ average structure of $\mathrm{BaTiO}_{3}$ (isomorphous to the equivalent high temperature tetragonal phase of $\mathrm{KNbO}_{3}$, see [6]) shown in Fig. 1(b) for example, confirms the existence of $\mathbf{G} \pm\left(\begin{array}{lll}1 & 0 & 0\end{array}\right)^{*}$ and $\mathbf{G} \pm\left(\begin{array}{lll}0 & 1 & 0\end{array}\right)^{*}$ sheets of diffuse intensity in this tetragonal phase while the absence of a $\mathbf{G} \pm\left(\begin{array}{lll}0 & 0 & 1\end{array}\right)^{*}$ sheet of diffuse intensity is consistent with the presence of long range polar order along the [ $\left[\begin{array}{lll}0 & 0 & 1\end{array}\right]$ direction (see also [6]).

Changes in the correlation structure of this first, triply degenerate, degree of freedom (there are three separate $\left\langle\begin{array}{lll}0 & 0 & 1\end{array}\right\rangle_{p}$ directions), from short to long range order (and from dynamic to static $[9,10])$, are thus the order parameters responsible for the 'cubic to tetragonal' (C-T) and 'tetragonal to orthorhombic' (T-O) phase transitions (horizontal with composition; see, e.g., the original phase diagram of Ahtee and Glazer [11]) at $\sim 400$ and $\sim 200{ }^{\circ} \mathrm{C}$, respectively, in the KNN $x$ system as well as the lower than room temperature 'orthorhombic to rhombohedral' (O-R) phase transition. Note that Comes et al. [6-8] first showed the existence of three $\mathbf{G} \pm\left\{\begin{array}{lll}0 & 0 & 1\end{array}\right\}^{*}$ sheets of diffuse intensity in the high temperature cubic paraelectric phase of $\mathrm{KNbO}_{3}$, two $\mathbf{G} \pm\left\{\begin{array}{lll}0 & 0 & 1\end{array}\right\}^{*}$ sheets of diffuse intensity in the tetragonal ferroelectric phase, one $\mathbf{G} \pm\left\{\begin{array}{lll}0 & 0 & 1\end{array}\right\}^{*}$ sheet of diffuse intensity in the orthorhombic phase and no $\mathbf{G} \pm\left\{\begin{array}{lll}0 & 0 & 1\end{array}\right\}^{*}$ sheets of diffuse intensity in the low temperature, fully ordered, rhombohedral ferroelectric phase.

At room temperature in the KNN $x$ system, two of the three possible NPR degrees of freedom are locked-in giving rise to long range ferroelectric order in the plane perpendicular to a parent perovskite $\left\langle\begin{array}{llll}0 & 0 & 1\end{array}\right\rangle_{p}$ direction (often taken to be the $\left[\begin{array}{lll}0 & 1 & 0\end{array}\right]_{p}$ direction in the case of the KNN $x$ system $[5,11,12])$. Long range ferroelectric order is then established along both the $\left[\begin{array}{lll}1 & 0 & 0\end{array}\right]_{p}$ and $\left[\begin{array}{lll}0 & 0 & 1\end{array}\right]_{p}$ directions. Under the assumption that the amplitude as well as the displacement eigenvectors of these two locked-in basis modes are the same, the direction of the macroscopic spontaneous polarisation is thus necessarily along a parent perovskite $\left\langle\begin{array}{llll}1 & 0 & 1\end{array}\right\rangle_{p}$ real space direction, as is indeed found experimentally in the case of the end-member $\mathrm{KNbO}_{3}(x=0)$ structure [6-8] (see Fig. 2(a); again the various off-centre displacements involved have each been increased by a factor of three to make them more apparent). This pattern of off-centre displacements responsible for macroscopic polarisation in the case of KNN $x=0$ might then quite reasonably be expected to remain largely unchanged across the entire KNN $x$ phase diagram unless there is an unexpectedly strong coupling between this ferroelectric ordering and the second order parameter, octahedral tilt rotation. 
a

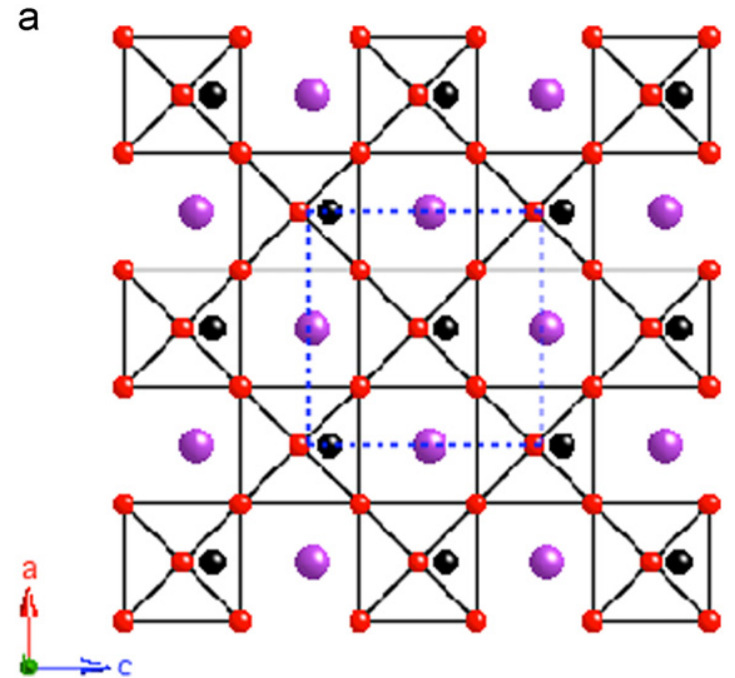

$\mathrm{b}$

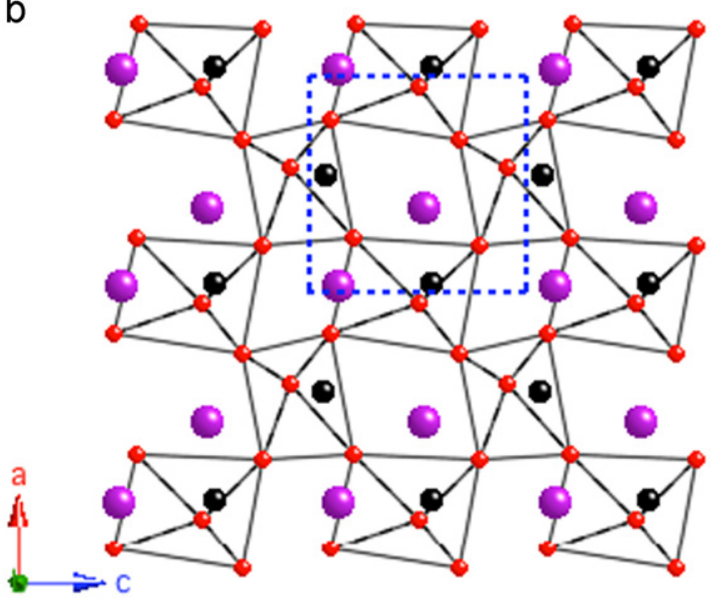

Fig. 2. (a) The Bmm2 ( $\left.\sqrt{ } 2 \times 1 \times \sqrt{ } 2, \mathbf{a}=\mathbf{a}_{p}+\mathbf{c}_{p}, \mathbf{b}=\mathbf{b}_{p}, \mathbf{c}=-\mathbf{a}_{p}+\mathbf{c}_{p}\right)$ setting [19] of $\mathrm{KNbO}_{3}$ with displacements exaggerated by a factor of 3 and (b) the literature monoclinic P1m1 structure of KNN 30 [17], again with off-centre displacements increased by a factor of 3 .

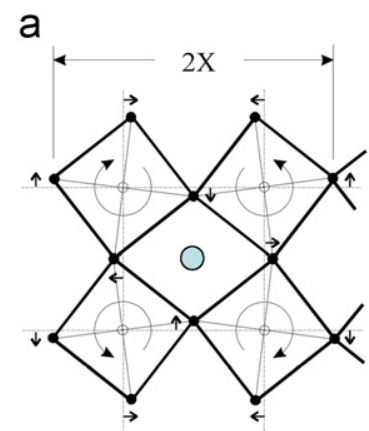

(001) b

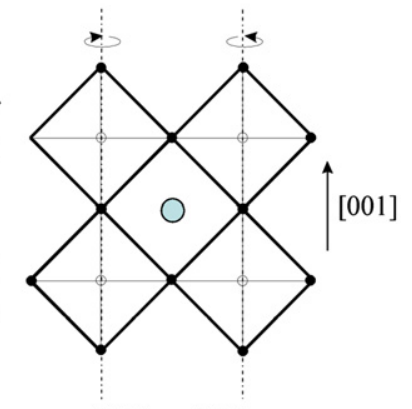

(100) or $(010)$
Fig. 3. (a) Octahedral tilting, around one of the three $\langle 001\rangle$ p directions viewed along that axis and (b) the same octahedral tilting viewed along an orthogonal $\left\langle\begin{array}{lll}0 & 0 & 1\end{array}\right\rangle_{p}$ axis with the clear doubling along those axes apparent.

The second such triply degenerate degree of freedom is the extent and pattern of octahedral tilting, again around one or more of the three $\left\langle\begin{array}{llll}0 & 0 & 1\end{array}\right\rangle_{p}$ directions (see Fig. 3), induced by the systematic introduction of the rather smaller, and hence significantly under-bonded, $\mathrm{Na}^{+}$ions into the perovskite A sites of the originally unrotated but ferroelectric, $A m m 2, \mathrm{KNbO}_{3}$ structure type on reducing $x$ from unity. According to the phase diagram of Ahtee and Glazer [11], no long range octahedral rotation occurs in the KNN $x$ system until $x$ is reduced to $\sim 0.48$ when a $\boldsymbol{b}^{+}$ octahedral tilt rotation pattern is reported to set in. (Note that the superscript + here implies that the amplitude of rotation around the $\mathbf{b}_{p}$ axis is the same from one ( $\left.\begin{array}{lll}0 & 1 & 0\end{array}\right)$ layer to the next and necessitates the existence of $\mathbf{G} \pm{ }^{1} /{ }_{2}\left[\begin{array}{lll}1 & 0 & 1\end{array}\right]_{p}{ }^{*}$ satellite reflections). A recently updated phase diagram [12] suggests that the onset of $\boldsymbol{b}^{+}$tilting does not occur until an even lower $x$ value of $\sim 0.4$. $\boldsymbol{b}^{+}$ tilting is then reported to occur from $x=0.4$ down to $\sim 0.2$, below which $\boldsymbol{a}^{-}, \boldsymbol{b}^{+}$and $\boldsymbol{c}^{-}$tilting are all reported to occur. (The superscript - here implies that the amplitude of rotation around the $\mathbf{a}_{p} \& \mathbf{c}_{p}$ axes reverse in sign from one ( $\left.\begin{array}{lll}0 & 1 & 0\end{array}\right)$ layer to the next and necessitates the existence of $\mathbf{G} \pm{ }^{1} / 2\left[\begin{array}{lll}1 & 1 & 1\end{array}\right]_{p}{ }^{*}$ satellite reflections).

As for the ferroelectric degree of freedom, this octahedral tilt degree of freedom can be both long range, as well as short range, ordered. (The latter fact is again not widely appreciated, given that most structural work performed on KNN $x$ to date has consisted only of conventional powder diffraction using neutrons [5] and/or X-rays [11, 12], techniques which are not particularly sensitive to such short range order). Octahedral rotation around $\left[\begin{array}{lll}0 & 0 & 1\end{array}\right]_{p}$, for example, necessarily gives rise to a correlated $2 x$ repeat along each of the orthogonal $\left[\begin{array}{lll}1 & 0 & 0\end{array}\right]$ and $\left[\begin{array}{lll}0 & 1 & 0\end{array}\right]$ directions (see Fig. 3), i.e., the displacive modulation associated with octahedral rotation is necessarily characterised by a modulation wave-vector of $[1 / 2,1 / 2, \xi]^{*}$ ( $\xi$ not fixed) type. Because the axis of rotation is perpendicular to the $\left(\begin{array}{lll}0 & 0 & 1\end{array}\right)$ plane, however, there is no necessary correlated coupling of the magnitude or sign of this rotation along the [ $\left[\begin{array}{lll}0 & 0 & 1\end{array}\right]$ direction from one such $\left(\begin{array}{lll}0 & 0 & 1\end{array}\right)$ type plane to the next, at least as far as the $\mathrm{NbO}_{6}$ octahedral array is concerned. In reciprocal space, octahedral tilt disorder manifests itself in the presence of rods of diffuse intensity parallel to $\left[\begin{array}{lll}1 & 0 & 0\end{array}\right]_{p}{ }^{*},\left[\begin{array}{lll}0 & 1 & 0\end{array}\right]_{p}{ }^{*}$ and $\left[\begin{array}{lll}0 & 0 & 1\end{array}\right]_{p}{ }^{*}$ associated with modulation wave vectors of $\langle 1 / 2,1 / 2, \xi\rangle^{*}$ type. This apparently 'disordered' octahedral tilting is particularly likely to occur in the current case where over-bonded $\mathrm{K}^{+}$and under-bonded $\mathrm{Na}^{+}$simultaneously occupy the same perovskite $\mathrm{A}$ site position and thus require different degrees of local octahedral tilting to satisfy as far as possible their local bonding requirements. Note, however, that streaking of the type $\mathrm{G}_{p} \pm\left[\xi, 1 /{ }_{2}, 1 / 2\right]_{p}{ }^{*}$ has previously been observed even in pure $\mathrm{NaNbO}_{3}$ near a phase transition at $641 \mathrm{~K}$, and reported as a critical phenomenon by Denoyer et al. [13].

Very few diffuse scattering investigations have been performed on this KNN $x$ system since the pioneering work of Comes et al. on $\mathrm{KNbO}_{3}$ [6-8]. Given the above discussion and the reported phase diagrams $[11,12]$, one would therefore expect only a single sheet of diffuse intensity perpendicular to $\mathbf{b}_{p}{ }^{*}$ from a single domain of the orthorhombic/monoclinic room temperature phase, by analogy with the known behaviour of $\mathrm{BaTiO}_{3}$ [7], $\mathrm{KNbO}_{3}$ [6,7] and $\mathrm{NaNbO}_{3}$ [13]. This is indeed the case, as we have previously reported [14] for one particular composition, KNN 46. The absence of similar diffuse sheets of intensity perpendicular to $\mathbf{a}_{p}^{*}$ and $\mathbf{c}_{p}^{*}$ thus implies the existence of long range ferroelectric order along two of the three parent perovskite, $\left\langle\begin{array}{lll}0 & 0 & 1\end{array}\right\rangle_{p}$ directions but disordered NPR's along the third, in this case the $\left[\begin{array}{lll}0 & 1 & 0\end{array}\right]_{p}$ direction (see [6,7]). In addition to this characteristic type of structured diffuse scattering associated with the first order parameter, however, there is also structured diffuse scattering associated with the second order parameter in the form of $\mathbf{G} \pm[1 / 2,1 / 2, \xi]_{p}{ }^{*}$ rods of diffuse intensity ( $\xi$ essentially continuous).

This report seeks to expand on the complex order/disorder and its behaviour across the KNN $x$ phase diagram using the above theoretical framework, as well as its possible implications for structure determination and properties. 


\section{Experimental}

Synthesis was performed by solid-state reaction for samples of composition $x=0.15,0.21,0.35,0.46$ and 0.75 . Starting reagents were $\mathrm{K}_{2} \mathrm{CO}_{3}, \mathrm{Na}_{2} \mathrm{CO}_{3}$ and $\mathrm{Nb}_{2} \mathrm{O}_{5}$. Calcining and sintering were performed in a muffle furnace. Mixing and homogenising of reagents was performed in a planetary ball mill, using yttrium stabilised zirconia balls in polymeric tanks. The carbonates and oxides were ball milled in stoichiometric ratios for $12 \mathrm{~h}$ under ethanol followed by calcining at $900{ }^{\circ} \mathrm{C}$ for $5 \mathrm{~h}$ to drive off carbon dioxide and begin the reaction to form KNN. The ball milling and calcination at $900{ }^{\circ} \mathrm{C}$ was then repeated to ensure homogeneity and the complete decomposition of the starting reagents. Finally, the powders were ball milled again for $12 \mathrm{~h}$ under ethanol and pressed into $20 \mathrm{~mm}$ diameter pellets under $8 \mathrm{t}$ of applied force in a uniaxial press followed by sintering in a bed of loose powder of the same composition on Pt foil, in a lidded alumina crucible for $4 \mathrm{~h}$ in the temperature range of $1030{ }^{\circ} \mathrm{C}$ for KNN, $x=0.15$ to $1110{ }^{\circ} \mathrm{C}$ for $\mathrm{KNN}, x=0.75$. The $x=0.46$ sample was prepared by sol-gel synthesis for better stoichiometric control. The reaction conditions are as described in our previous paper [14].

X-ray powder diffraction (XRPD) work was performed with a Guinier-Hägg camera using monochromatic $\mathrm{Cu} K_{\alpha 1}$ radiation, with pure silicon (Sietronics GD\#1) [15] added as an internal standard. All samples were successfully prepared as single-phase pellets, with excellent crystallinity, as determined from powder XRD. Electron diffraction patterns (EDP's) were obtained using Philips EM 430 and Philips CM 30 Transmission Electron Microscopes (TEMs) operating at $300 \mathrm{kV}$ on crushed sample powders dispersed onto holey carbon coated, copper grids.

Bond valence sum calculations were performed by creating a model using the perovskite octahedral tilting programme "POTATO" [16] and the lattice parameters from the CIF file for KNN with $x=0.3$ (ICSD entry 173740) [17] and then using this as an input to the bond valence sum programme "EUTAX" [18].

\section{Results and discussion}

\subsection{Space group and structural considerations}

We begin by examining how the various room temperature phases reported for the KNN $x$ system (see the original phase diagram in [11] and the recently revised phase diagram given in [12]) are related to the archetypal $\mathrm{KNbO}_{3}$ end-member structure of this system. At room temperature, $\mathrm{KNbO}_{3}$ is typically reported to be in an orthorhombic Amm2 $\left(1 \times \sqrt{ } 2 \times \sqrt{ } 2=\mathbf{a}_{p}, \mathbf{b}=\mathbf{b}_{p}-\mathbf{c}_{p}\right.$, $\mathbf{c}=\mathbf{b}_{p}+\mathbf{c}_{p}$ ) structure [19], as shown schematically in an alternative Bmm2 $\left(\sqrt{ } 2 \times 1 \times \sqrt{ } 2, \mathbf{a}=\mathbf{a}_{p}+\mathbf{c}_{p}, \mathbf{b}=\mathbf{b}_{p}, \mathbf{c}=-\mathbf{a}_{p}+\mathbf{c}_{p}\right)$ setting in Fig. 2(a). In this phase, the $\mathrm{Nb}$ ions are constrained to be displaced only along the polar $\mathbf{c}=\mathbf{b}_{p}+\mathbf{c}_{p}$ direction and no octahedral rotation is allowed as a result of the strong over-bonding of the $\mathrm{K}^{+}$ions on the parent perovskite A site positions. Addition of the rather smaller, significantly under-bonded $\mathrm{Na}^{+}$ions, however, creates a driving force for local octahedral rotation (as well as for off-centre displacements of the $\mathrm{Na}^{+}$ions). Increasing the amount of $\mathrm{Na}^{+}$ions increases this driving force. The originally reported phase diagram suggests that there is no onset of long range ordered octahedral rotation until the concentration of $\mathrm{Na}^{+}$ions increases to $52 \%$ at $x=0.48$. This does not, however, rule out the possibility of short range ordered, local octahedral tilting (as is indeed shown to be the case below). The larger $\mathrm{K}^{+}$ions, however, are still significantly over-bonded and thus will still continue to oppose octahedral rotation so that any such local octahedral rotations will depend strongly on the local $\mathrm{Na} / \mathrm{K}$ distribution.
The addition of long range ordered $\boldsymbol{b}^{+}$octahedral tilting, as signified by the presence of sharp $\mathbf{G} \pm{ }^{1} / 2\left[\begin{array}{lll}1 & 0 & 1\end{array}\right]_{p}{ }^{*}$ satellite reflections, to the pre-existing off-centred ferroelectric ordering (see Fig. 2(a) at around $x=0.48$ then necessarily lowers the original Amm2 space group symmetry. Before considering the effect of adding such octahedral rotation around $\mathbf{b}_{p}$ to the preexisting ferroelectric ordering along $\left[\begin{array}{llll}1 & 0 & 1\end{array}\right]_{p}$, we first change the setting of the orthorhombic Amm2 structure type to orthorhombic Bmm2 (as discussed above, see Fig. 2(a)) so as to be consistent with the unique $b$ axis convention normally used for the KNN system for $0.2<x<0.48[11,12]$. Using standard group-subgroup relationships, it is then not difficult to show that adding $\boldsymbol{b}^{+}$ octahedral rotation to ferroelectric displacement along [ $\left[\begin{array}{lll}1 & 0 & 1\end{array}\right]_{p}$ necessarily leads to a resultant orthorhombic $P c m 2{ }_{1}$ space group symmetry with the same resultant unit cell and with the spontaneous polarisation still aligned along the $c$ direction as before. We recently found just such a space group symmetry and unit cell for KNN, $x=0.46$ from electron diffraction data [14].

Traditionally, however, this phase has always been refined using the same unit cell but assuming monoclinic $P 1 \mathrm{~m} 1$ space group symmetry [11,12]. Baker et al. [17], for example, have recently reported an $a=5.64304(5) \AA, \quad b=3.93188(2) \AA, \quad c=5.61260(5) \AA$, $\beta=89.9136(6)^{\circ}$ structure for KNN 30 in space group $P 1 m 1$ (shown in Fig. 2(b); again the various off-centre displacements involved have each been increased by a factor of three to make them more apparent). This lowering of space group symmetry from orthorhombic $P c m 2$ to monoclinic $P 1 \mathrm{~m} 1$ has lead to a resultant structure with quite significantly distorted $\mathrm{NbO}_{6}$ octahedra and one where it is rather difficult, if not impossible, to identify the off-centre displacements primarily responsible for ferroelectricity or to relate these offcentre ferroelectric displacements to those characteristic of $\mathrm{KNbO}_{3}$, the end-member $x=1$ compound ( $c f$., for example, Fig. 2(b) with (a)). Such a large change in the displacement eigenvectors of the primary $\mathbf{q}=\mathbf{0}$, ferroelectric basis modes arising from the onset of weak octahedral tilting would be highly irregular from a group theoretical point of view and also seems highly unlikely from a crystal chemical point of view. This is also compatible with theoretical calculations (see, e.g., [20]).

Carrying on to lower values of $x$, we now investigate what happens to the proposed $\sqrt{ } 2 \times 1 \times \sqrt{ } 2$ unit cell and $P c m 2_{1}$ space group symmetry (see [14]), below the octahedral tilting phase transition around $x \sim 0.17[11,12]$ associated with the onset of additional Glazer ' - ' tilting around the $\mathbf{c}=\mathbf{a}_{p}+\mathbf{c}_{p}$ direction of the proposed $P c m 2_{1}$ structure. In order to do this, we take the previously described $P c m 22_{1}$ structure and introduce an additional Glazer ' - ' rotation around $\left[\begin{array}{lll}1 & 0 & 1\end{array}\right]_{p}$. Doing this gives rise to the additional $\mathbf{G} \pm{ }^{1} /{ }_{2}\left[\begin{array}{lll}1 & 1 & 1\end{array}\right]_{p}{ }^{*}$ reflections experimentally observed and to an $a^{-} b^{+} a^{-}$octahedral tilt system (equivalent to an $a^{-} b^{+} c^{-}$tilt system because of the pre-existing orthorhombic symmetry). The additional tilt changes the initial $\sqrt{ } 2 \times 1 \times \sqrt{ } 2$ supercell to a $\sqrt{ } 2 \times 2 \times \sqrt{ } 2$ supercell. The space group symmetry of the new doubled superstructure, however, remains the same, i.e., $P c m 2_{1}$. Experimentally, the reported unit cell refined from powder data for this phase is (ICSD entry 28045) [21] $a=7.8782(7) \AA, \quad b=7.7920(7) \AA$, $c=7.8632(7) \AA, \alpha=90^{\circ}, \beta=90.479(4)^{\circ}, \gamma=90^{\circ}$ which in turn is equivalent to an $a=5.5886 \AA, b=7.7920 \AA, c=5.5421 \AA, \alpha=90^{\circ}$, $\beta=89.891^{\circ}, \gamma=90^{\circ}$ in the $\sqrt{2} \times 2 \times \sqrt{ } 2$ supercell setting. This is, as mentioned in the original article, "very (... very ...) nearly orthorhombic" [21]!

The above analysis strongly suggests that the apparent monoclinic symmetry that has been reported from powder diffraction data for both of these low $\times$ phases may well be an artefact that comes from some sort of deficiency in either the powder diffraction data itself or the structural model being refined from this powder data. We believe this is likely to arise from the neglect of inherent displacive disorder as discussed above and the influence 
of this disorder on the reflections observed in a powder diffraction pattern. In order to investigate this inherent displacive disorder, electron diffraction as a function of composition is the obvious tool of choice.

\subsection{Electron diffraction results}

A systematic investigation of the reciprocal space of KNN $x$ as a function of composition in each of the separate phase fields identified in $[11,12]$ is shown in Figs. 4 and 5. As above, we start with the unrotated but ferroelectric, high $x$ samples and then consider the various octahedrally rotated phases which occur when $x$ is reduced systematically. For comparison purposes, we will keep the unique ferroelectrically disordered $\left\langle\begin{array}{lll}0 & 0 & 1\end{array}\right\rangle_{p}$ axis as the $\left[\begin{array}{lll}0 & 1 & 0\end{array}\right]_{p}$ axis throughout. For the sample with $x=0.75$, in the middle of the unrotated solid solution phase of which $\mathrm{KNbO}_{3}$ itself is one end-member, there are no sharp superlattice reflections in addition to the parent perovskite sub-structure reflections $\mathbf{G}$ (see Fig. 4). This is as expected from the refined $A m m 2$ structure of the end-member $\mathrm{KNbO}_{3}$, in the $\mathrm{Bmm} 2$ setting a



b



Fig. 4. (a) Close to $\left[\begin{array}{lll}0 & 0 & 1\end{array}\right]_{p}$ and (b) $\left[\begin{array}{lll}3 & 1 & 0\end{array}\right]_{p}$ zone axis EDPs of $\mathrm{K}_{0.75} \mathrm{Na}_{0.25} \mathrm{NbO}_{3}$, indexed according to both the Bmm2 space group (no subscripts) and the parent perovskite subcell (denoted by a subscript $p$ ). Chevron-headed arrows indicate transverse-polarised planes of diffuse scattering perpendicular to [0 10$]_{p}{ }^{*}$. Solid-headed arrows indicate "spots", associated with diffuse streaking of the type $\mathbf{G} \pm[\xi, 1 / 2,1 / 2]_{p}{ }^{*}$ and $\mathbf{G} \pm[1 / 2,1 / 2, \xi]_{p}{ }^{*}$.
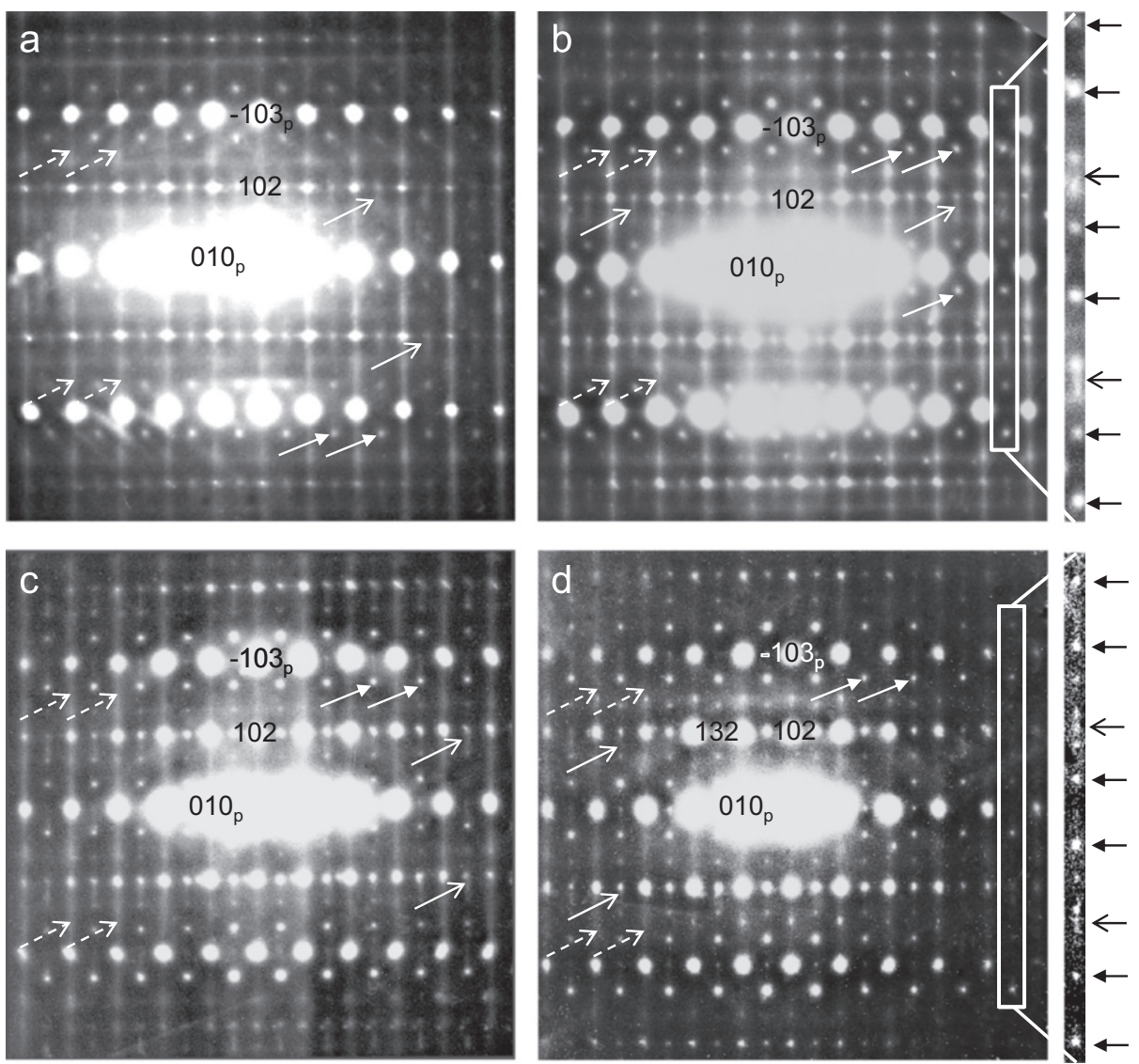

Fig. 5. $[-3,0 .-1] p$ zone axis EDPs of (a) $\mathrm{K}_{0.46} \mathrm{Na}_{0.54} \mathrm{NbO}_{3}$, (b) $\mathrm{K}_{0.35} \mathrm{Na}_{0.65} \mathrm{NbO}_{3}$, (c) $\mathrm{K}_{0.21} \mathrm{Na}_{0.79} \mathrm{NbO}_{3}$ and (d) $\mathrm{K}_{0.15} \mathrm{Na}_{0.85} \mathrm{NbO}_{3}$ indexed according to both the relevant $P c m 2{ }_{1}$ space groups (no subscripts) and the parent perovskite subcell (denoted by a subscript $p$ ). Arrows with dashed tails indicate transverse-polarised planes of diffuse

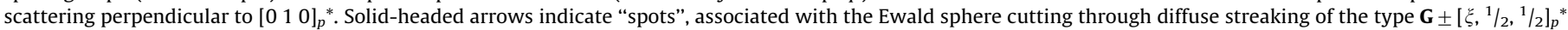
and $\mathbf{G} \pm[1 / 2,1 / 2, \xi]_{p}{ }^{*}$. Chevron-headed arrows with solid tails indicate diffuse streaking the same type, but with the streak seen in plane. 
$\left(\sqrt{ } 2 \times 1 \times \sqrt{ } 2, \quad \mathbf{a}=\mathbf{a}_{p}+\mathbf{c}_{p}, \quad \mathbf{b}=\mathbf{b}_{p}, \quad \mathbf{c}=-\mathbf{a}_{p}+\mathbf{c}_{p} ; \quad \mathbf{a}^{*}={ }^{1} / 2 \quad\left[\begin{array}{lll}1 & 0 & 1\end{array}\right]_{p}{ }^{*}\right.$, $\left.\mathbf{b}^{*}=\left[\begin{array}{lll}0 & 1 & 0\end{array}\right]_{p}^{*}, \mathbf{c}^{*}={ }^{1} / 2[-1,0,1]_{p}{ }^{*}\right)$.

There are, however, quite clear additional diffuse features in the form of $\mathbf{G} \pm\left(\begin{array}{lll}0 & 1 & 0\end{array}\right)_{p}{ }^{*}$ sheets of diffuse intensity (see e.g., the transverse polarised, diffuse streaking, indicated by arrows with dashed tails, running through the parent perovskite Bragg reflections along $\left[\begin{array}{lll}0 & 0 & 1\end{array}\right]_{p}^{*}$ (perpendicular to $\left[\begin{array}{lll}0 & 1 & 0\end{array}\right]_{p}$ ) but not $\left[\begin{array}{lll}0 & 1 & 0\end{array}\right]_{p}^{*}$ in Fig. 4(a) and

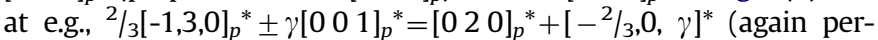
pendicular to $\left.\left[\begin{array}{lll}0 & 1 & 0\end{array}\right]_{p}\right)$ in Fig. 4(b). In addition to this $\mathbf{G} \pm\left(\begin{array}{lll}0 & 1 & 0\end{array}\right)_{p}{ }^{*}$ sheet of diffuse intensity, there are also clear blobs of diffuse intensity at $\mathbf{G} \pm\left[0,{ }^{1} / 2,{ }^{1} / 2\right]_{p}{ }^{*}$ (solid headed arrowed in Fig. 4(a) and at $\mathbf{G} \pm\left[1 / 2,-{ }^{3} / 2,1\right]_{p}{ }^{*}=\mathbf{G} \pm[1 / 2,1 / 2,0]_{p}{ }^{*}$ and $\mathbf{G} \pm{ }^{1} / 6[-1,3,3]_{p}{ }^{*}=\mathbf{G} \pm$ $\left[{ }^{1} / 6,1 / 2,1 / 2\right]_{p}{ }^{*}$ (solid headed arrows in Fig. 4(b) corresponding to cuts through essentially continuous $\mathbf{G} \pm\left[\xi,{ }^{1} / 2,{ }^{1} / 2\right]_{p}{ }^{*}$ and $\mathbf{G} \pm[1 / 2,1 / 2, \xi]_{p}{ }^{*}$ rods of diffuse intensity. Clearly there is considerable octahedral tilt disorder in this KNN 75 sample. These rods of diffuse intensity will be discussed in more detail below as they are common across the entire KNN $x$ composition range.

Note that multiple scattering can re-distribute the intensity of the observed diffuse intensity from e.g., $\mathbf{G}_{1} \pm\left[\xi, 1 / 2,{ }^{1} / 2\right]_{p}{ }^{*}$ to $\mathbf{G}_{\mathbf{2}} \pm[\xi, 1 / 2,1 / 2]_{p}{ }^{*}$ but it can not create any arbitrary $\mathbf{G} \pm[\xi, 1 / 2$, $1 / 2]_{p}{ }^{*}$ reflection in the first place (for more details of the effect of multiple scattering on diffuse distributions, see [22]).

Fig. 5 shows the same $\left[\begin{array}{lll}3 & 0 & 1\end{array}\right]_{p}=[2,0,-1]$ zone axis EDP for the (a) $x=0.46$, (b) $x=0.35$, (c) $x=0.21$ and (d) $x=0.15$ samples. Note that the $x=0.46$ and 0.35 samples are nominally in the $L\left(a^{0} b^{+} a^{0}\right)$ phase region, the $x=0.21$ sample in the $\mathrm{K}\left(a^{-} b^{+} c^{0}\right)$ phase region and the $x=0.15$ sample in the $\mathrm{Q}\left(a^{-} b^{+} c^{-}\right)$phase region according to the original phase diagram of Ahtee and Glazer [11]. The recent partially revised phase diagram of Baker et al. [12] removes the phase boundary from $\mathrm{L}\left(a^{0} b^{+} c^{0}\right)$ to $\mathrm{K}\left(a^{-} b^{+} c^{0}\right)$ at $x \sim 0.32$, suggesting that the $\mathrm{K}$ phase does not exist.

As is well known, $b^{+}$tilting is signified by the presence of sharp $\mathbf{G} \pm{ }^{1} / 2\left[\begin{array}{lll}1 & 0 & 1\end{array}\right]_{p}{ }^{*}$ satellite reflections such as, e.g., the reflection labelled $\left[\begin{array}{lll}1 & 0 & 2\end{array}\right]^{*}\left({ }^{1} / 2\left[\begin{array}{lll}-1 & 0 & 3\end{array}\right]_{p}{ }^{*}=\left[\begin{array}{lll}-1 & 0 & 1\end{array}\right]_{p}{ }^{*}+{ }^{1} / 2\left[\begin{array}{lll}1 & 0 & 1\end{array}\right]_{p}{ }^{*}\right)$ in all EDP's of Fig. 5 [23]. Note that the intensity of these satellite reflections systematically increases with increasing $\mathrm{Na}^{+}$concentration, as would be expected crystal chemically. The onset of 'tilting, on the other hand, implies the presence of sharp $\mathbf{G} \pm$ $1 / 2 \quad\left[\begin{array}{lll}1 & 1 & 1\end{array}\right]_{p}{ }^{*}$ satellite reflections such as, e.g., the reflection labelled $\left[\begin{array}{lll}1 & 3 & 2\end{array}\right]^{*}\left(={ }^{1} / 2\left[\begin{array}{lll}-1 & 3 & 3\end{array}\right]_{p}{ }^{*}=\left[\begin{array}{lll}-1 & 1 & 1\end{array}\right]_{p}{ }^{*}+{ }^{1} / 2 \quad\left[\begin{array}{lll}1 & 1 & 1\end{array}\right]_{p}{ }^{*}\right)$ in Fig. 5(d). This is as expected given that the $Q\left(a^{-} b^{+} c^{-}\right)$phase contains "-' tilting. Note that weak but quite sharp satellite reflections also exist at the $\mathbf{G} \pm{ }^{1} / 2\left[\begin{array}{lll}0 & 1 & 0\end{array}\right]_{p}{ }^{*}$ positions of reciprocal space in Fig. 5(d), consistent with being induced by co-existing $\mathbf{G} \pm{ }^{1} /{ }_{2}\left[\begin{array}{lll}1 & 1 & 1\end{array}\right]_{p}{ }^{*}$ and $\mathbf{G} \pm{ }^{1} / 2\left[\begin{array}{lll}1 & 0 & 1\end{array}\right]_{p}{ }^{*}$ type satellite reflections in this $Q\left(a^{-} b^{+} c^{-}\right)$phase. There is also some much weaker indication of the existence of $\mathbf{G} \pm{ }^{1} / 2 \quad\left[\begin{array}{lll}0 & 1 & 0\end{array}\right]_{p}{ }^{*}$ 'satellite reflections' in Fig. 5(c) as well, suggesting that a "-' tilt may also exist in the KNN 21 sample and that the recently discarded " $K$ " phase (see [12]) might well actually exist. On the other hand, the $\mathbf{G} \pm{ }^{1} /{ }_{2}\left[\begin{array}{lll}1 & 1 & 1\end{array}\right]_{p}{ }^{*}$ 'reflections' also appear to be present in Fig. 5(a) and (b) where they clearly should not be present according to the phase diagrams reported in [11] and [12].

The resolution of this apparent conflict is to note that the three $\mathbf{G} \pm\left\langle 1 / 2,{ }^{1} / 2, \xi\right\rangle_{p}{ }^{*}$ rods of diffuse intensity (indicative of octahedral tilt disorder, as mentioned above) intersect at the $\mathbf{G} \pm$ ${ }^{1 / 2}\left[\begin{array}{lll}1 & 1 & 1\end{array}\right]_{p}{ }^{*}$ positions of reciprocal space and will thus give rise to apparent 'satellite reflections' as observed experimentally over the entire KNN $x$ system (see, e.g., Figs. 4(b), 5(a)-(c)). The fact that the diffuse 'tails' to these 'satellite reflections', denoted by chevron-headed arrows with solid tails in Fig. 5, always appear on one particular side of the local $\mathbf{G} \pm{ }^{1} /{ }_{2}\left[\begin{array}{lll}1 & 1 & 1\end{array}\right]_{p}{ }^{*}$ positions of reciprocal space is characteristic of the intersection between the 1-D rod shape of the diffuse and the slight upwards curvature of the Ewald sphere in the case of electron diffraction, as shown in
Fig. 6. What is observed experimentally in any one EDP clearly depends upon how the Ewald sphere cuts through the 1-D rods of diffuse intensity. Consider, for example, the $\left[0,1 / 2,-{ }^{1} / 2\right]_{p}{ }^{*}$ point of reciprocal space in Fig. 6 . The $\left[\xi, 1 / 2,{ }_{1}^{1} / 2\right]_{p}{ }^{*} \operatorname{rod}$ of 1 -D diffuse is shown in Fig. 6 as the black line passing through this point. Because this rod of diffuse cuts through the Ewald sphere very quickly it gives rise to what appears to be sharp 'satellite reflections' (indicated with solid-headed arrows in Fig. 5(b) and (d) at the $\mathbf{G} \pm\left[-{ }^{1} / 6,1 / 2,{ }^{1} / 2\right]_{p}{ }^{*}$ regions of reciprocal space. 'Reflections' of this type are absolutely symptomatic of octahedral tilt disorder and exist right across the KNN $x$ system ( $c f$. , e.g., the same apparent 'reflections' in Fig. 4(b) and in every EDP in Fig. 5. Clearly the detection of intensity at the $\mathbf{G} \pm{ }^{1} /{ }_{2}\left[\begin{array}{lll}1 & 0 & 1\end{array}\right]_{p}{ }^{*}$ and $\mathbf{G} \pm{ }^{1} / 2\left[\begin{array}{lll}1 & 1 & 1\end{array}\right]_{p}{ }^{*}$ positions of reciprocal space does not eliminate octahedral tilt disorder!

If this octahedral tilt disorder is sufficiently common along any one particular $\left\langle\begin{array}{llll}0 & 0 & 1\end{array}\right\rangle_{p}$ direction, the average magnitude of rotation will refine to zero in average structure refinements despite the likelihood of still having a significant amplitude in any one particular $\left\{\begin{array}{llll}0 & 0 & 1\end{array}\right\}_{p}$ layer. We believe that the local magnitude of these 'disordered' tilts can be quite significant and may be estimated from refined unit cell parameters. The parent perovskite unit cell parameters refined for $\mathrm{KNN}, x=0.3$, for example, are $a_{p}=3.99 \AA, b_{p}=3.93 \AA$ and $c_{p}=3.98 \AA$. Octahedral tilting around $\mathbf{b}_{p}$ would normally suggest that the magnitude of the $a$ and $c$ axes (as well as the corresponding $a_{p}$ and $c_{p}$ ) axes

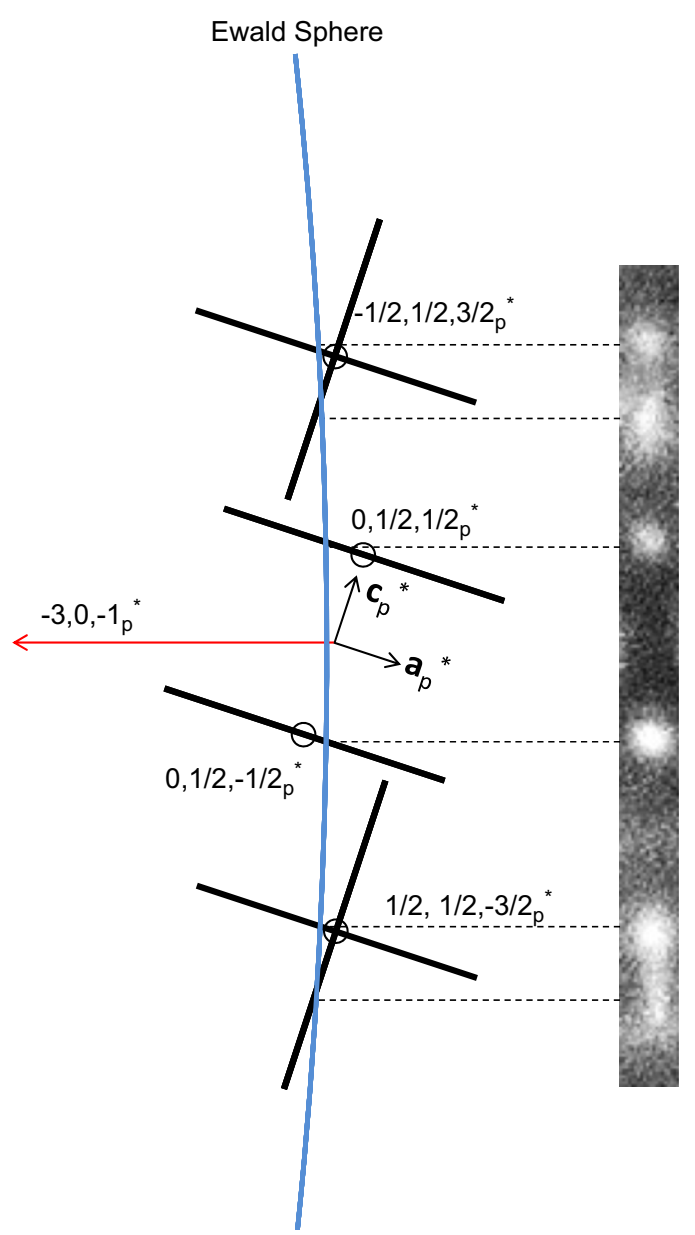

Fig. 6. An Ewald sphere construction showing the diffuse intensity arising from 1-D rod shaped diffuse giving both streaks and "spots" as a result of the relevant angle of intersection with the Ewald sphere. A highlight from Fig. 5(b) is shown for experimental comparison. 
should shrink relative to the $\mathbf{b}_{p}$ rotation axis of this phase (see, e.g., Fig. 3). The fact that the refined parent perovskite subcell dimensions do the exact opposite suggests that the disordered tilting around the parent $\mathbf{a}_{p}$ and $\mathbf{c}_{p}$ directions axes is in fact larger than the apparently fully ordered one around the $\mathbf{b}_{p}$ axis!

The local magnitude of this additional disordered local tilting can be estimated by assuming rigid $\mathrm{NbO}_{6}$ octahedra and tilting them from an ideal undistorted perovskite structure using the programme POTATO [16]. Due to the lack of obvious significant diffuse streaking in the $x=0.35$ sample along $\mathbf{b}_{p}^{*}$, the $\boldsymbol{b}^{+}$tilt angle was locked-in to the refined value of $3.4^{\circ}$ (see [17]). With this $\boldsymbol{b}^{+}$ tilt magnitude fixed, a unique solution for the magnitude of the disordered $\mathbf{a}_{p}$ and $\mathbf{c}_{p}$ tilts as well as a value for the $\mathrm{Nb}-\mathrm{O}$ bond length can be determined from the three refined lattice parameters. These disordered tilt magnitudes around the $\mathbf{a}_{p}$ and $\mathbf{c}_{p}$ axes refined to values $\sim 9$ to $10^{\circ}$ 's, or nearly three times that refined around $\mathbf{b}_{\boldsymbol{p}}$.

One possible explanation for the discrepancies between the space groups found from the current as well our previous examination at $x=0.46$ (see [14]) when compared to earlier neutron and X-ray diffraction analyses in the KNN $x$ system is the extensive $h k l$ dependent peak asymmetry that should be expected in high intensity powder diffraction data given the electron diffraction data presented in this contribution. The strong transverse polarised diffuse plane perpendicular to $\mathbf{b}_{p}{ }^{*}$ in Figs. 4 and 5, for example, must necessarily create peak asymmetry for parent $0 k 0$ and low $h$ and $l$, high $k, h k l$ relections towards high angle as well as broadening in many of the remaining parent peaks, although not in the $\mathbf{G} \pm{ }^{1} / 2\left[\begin{array}{llll}1 & 1 & 1\end{array}\right]_{p}{ }^{*}$ and $\mathbf{G} \pm^{1 / 2}\left[\begin{array}{lll}1 & 0 & 1\end{array}\right]_{p}{ }^{*}$ type satellite peaks. Likewise, the tilt-disorder related diffuse rod streaking will broaden satellite reflections relative to parent reflections but only along very specific directions of reciprocal space. The combination of such complex diffraction effects must create very significant problems in profile fitting from powder diffraction data that may well not be able to be modelled effectively via conventional Rietveld analysis. In such circumstances, the overall fit to high resolution powder data might well be improved by adding small deviations from orthorhombic to monoclinic symmetry, e.g., the approximately $0.1^{\circ} 2 \theta$ deviation away from metric orthorhombic symmetry reported for both monoclinic "Pm" phases in the KNN $x$ system [11,12]. A complement to lowering the symmetry is to introduce anisotropic thermal parameters for the oxygen atoms, which can be done when high quality data is available. This has been done recently by Zhang et al. [24] on the cubic paraelectric phase of PZT (which is in many ways analogous to KNN, being a ferroelectric perovskite) and clearly shows the existence of octahedral tilting above the Curie temperature in this material.

\subsection{Bond valence sum calculations}

Based on the experimental observations of diffuse scattering and their apparent conflict with previously published results, an examination of the effects of the proposed local structural distortions on the local crystal chemical environments was performed using bond valence sums. The impact of the different chemistry occurring in the locally $\mathrm{NaNbO}_{3}$-like and $\mathrm{KNbO}_{3}$-like sites in these KNN materials was considered and the interaction of these effects was found to be the most likely origin of structural disorder and the resulting diffuse scattering distributions.

It is known that bond valence sum analysis [18,25], in particular the use of the square of the so-called Global Instability Index $G_{i i}^{2}$, provides a very good approximation to the results of first principles energy calculations (see e.g., [26,27]), We thus use bond valence sum calculations of the likely local stability of KNN
Table 1

Bond valence sum totals, $0,+$ and - indicate Glazer tilts, while fe indicates B site ferroelectricity along $\left[\begin{array}{lll}1 & 1 & 1\end{array}\right]_{p}$ and $\mathrm{fe}_{2}$ indicates $\mathrm{A}$ and $\mathrm{B}$ site ferroelectricity also along [lll 111$]_{p}$. Tilt systems are arranged in order of increasing instability index.

\begin{tabular}{llll}
\hline Tilt system & $G_{i i}^{2}$ & Tilt system & $G_{i i}^{2}$ \\
\hline Element & $\mathrm{K} \downarrow$ & & $\mathrm{Na} \downarrow$ \\
$a^{0} b^{0} c^{0}$ & 0.224 & $a^{0} b^{0} c^{0}$ plus fe & 0.0068 \\
$a^{0} b^{0} c^{0}$ plus fe & 0.231 & $a^{0} b^{+} c^{0}$ plus fe & 0.0080 \\
$a^{0} b^{+} c^{0}$ & 0.236 & $a^{0} b^{0} c^{0}$ plus fe & 0.0131 \\
$a^{0} b^{+} c^{0}$ plus fe & 0.243 & $a^{0} b^{+} c^{0}$ plus fe & 0.0133 \\
Baker [14] & 0.395 & $a^{-} b^{+} c^{-}$plus fe & 0.0160 \\
$a^{+} b^{+} c^{-}$ & 0.554 & $a^{0} b^{0} c^{0}$ & 0.0165 \\
$a^{+} b^{+} c^{+}$ & 0.572 & $a^{0} b^{+} c^{0}$ & 0.0175 \\
$a^{-} b^{+} c^{-}$ & 0.584 & $a^{+} b^{+} c^{-}$plus fe & 0.0318 \\
$a^{+} b^{+} c^{-}$plus fe & 0.598 & $a^{+} b^{+} c^{+}$plus fe & 0.0337 \\
$a^{+} b^{+} c^{+}$plus fe & 0.617 & $B^{2}$ aker $[17]$ & 0.0471 \\
$a^{-} b^{+} c^{-}$plus fe & 0.618 & $a^{+} b^{+} c^{-}$ & 0.0945 \\
& & $a^{-} b^{+} c^{-}$ & 0.0955 \\
& & $a^{+} b^{+} c^{+}$ & 0.0993 \\
\hline
\end{tabular}

30 using a range of different models. The results are listed in Table 1.

Bond valence sum calculations performed in EUTAX (Table 1) show that the previously refined model for $x=0.3$ [17] results in a high squared global instability index $\left(G_{i i}^{2}\right.$, the RMS error in the apparent valences), particularly with $\mathrm{K}$ in the perovskite $\mathrm{A}$ site. An approximate overall $G_{i i}^{2}$ can be calculated by doing a linear sum of the $G_{i i}^{2}$ with $\mathrm{K}$ on the A site and with Na on the A site. The Baker et al. structure also includes heavily distorted octahedra, which may contribute to an increase in free energy due to effects not modelled in conventional bond valence sum calculations.

Bond valence sums, and hence $G_{i i}^{2}$, were calculated for various tilt configurations (listed in Table 1 in Glazer notation [23]) using tilt amplitudes refined from POTATO [16]. Where a tilt amplitude is listed as zero, the correponding oxygen atom degrees of freedom are not changed relative to the undistorted perovskite fractional co-ordinate positions. While this introduces some distortion of the octahedra, the calculated $G_{i i}^{2}$ are often significantly improved, particularly if the A site ion is heavily overbonded. Off-centre ferroelectric displacement of the $\mathrm{Nb}$ ion along $\left[\begin{array}{lll}1 & 1 & 1\end{array}\right]_{p}$ (fe) improves most bond valence sums. For some model structures with $\mathrm{Na}$ on the A site, a second ferroelectric displacement $\left(\mathrm{fe}_{2}\right)$ of the $\mathrm{Na}$ ion along [ $\left[\begin{array}{lll}1 & 1 & 1\end{array}\right]_{p}$ also gives some improvement.

A local ferroelectric displacement along [ $\left.\begin{array}{lll}1 & 1 & 1\end{array}\right]_{p}$, at least for the $\mathrm{Nb}$ perovskite $\mathrm{B}$ site ion, is expected as B site ferroelectric compounds $\left(\mathrm{KNbO}_{3}, \mathrm{BaTiO}_{3}\right.$, etc.) normally have a rhombohedral low temperature state. The observed structured diffuse scattering in such materials [6] suggests that local displacements are always along $\left\langle\begin{array}{lll}1 & 1 & 1\end{array}\right\rangle_{p}$. It is clear from the behaviour of $G_{i i}^{2}$ in Table 1 that model structures with $\mathrm{K}$ on the A site have significantly lower energy when no tilts are present around the $\mathbf{a}_{p}$ and $\mathbf{c}_{p}$ axes, while the structures with $\mathrm{Na}$ on the A site are relatively stable for a variety of tilt configurations. The lowest $G_{i i}^{2}$ for a model structure only allowing $\boldsymbol{b}^{+}$tilts fixed to the value refined by Baker et al. [17] (model 1) is given by:

$$
\begin{aligned}
G_{i i}^{2}= & 0.0080 \times 0.7\left(N a\left[a^{0} b^{+} c^{0} f e_{2}\right]\right)+0.2362 \\
& \times 0.3\left(K\left[a^{0} b^{+} c^{0}\right]\right)=0.0765
\end{aligned}
$$

While the addition of ferroelectric displacements to the model structure with $\mathrm{K}$ on the perovskite $\mathrm{A}$ site and addition of Glazer tilts to the model structure with $\mathrm{Na}$ on the perovskite A site (model 2) yields a minor change to:

$$
G_{i i}^{2}=0.0160 \times 0.7\left(\mathrm{Na}\left[a^{-} b^{+} c^{-} \mathrm{fe}\right]\right)+0.2427 \times 0.3\left(\mathrm{~K}\left[a^{0} b^{+} c^{0} \mathrm{fe}\right]\right)=
$$
0.0840 
Compare this with the previously refined structure $G_{i i}^{2}$ value for Baker et al. [17] of:

$G_{i i}^{2}=0.0471 \times 0.7+0.3955 \times 0.3=0.1516$

The decrease/improvement in $G_{i i}^{2}$ of $45-50 \%$ suggests a significantly more stable local structure.

The $a^{-} b^{+} c^{-}$tilt configuration in the case where $\mathrm{Na}$ is on the perovskite A site corresponds to tilt angles of $9.71^{\circ}$ around $\mathbf{a}_{p}$ and $9.57^{\circ}$ around $\mathbf{c}_{p}$ in order to match the literature lattice parameter values. As discussed above, these local rotation angles are significantly higher than the average $3.4^{\circ}$ rotation angle refined around the $\mathbf{b}_{p}$ axis. The slightly higher value of $G_{i i}^{2}$ for model 2 relative to model 1 may be compensated for due to smaller octahedral distortion and through compatibility with adjacent $\mathrm{Na}$ cell ferroelectricity.

The substantial difference in acceptable tilting environments for $\mathrm{K}$ and $\mathrm{Na}$ on the local scale may lead to structures that are likely to involve some degree of layered ordering of the $\mathrm{Na}$ and $\mathrm{K}$ ions, as octahedral tilting necessarily constrains whole layers to one rigid rotation angle. No clear evidence of A site ordering has been reported in the KNN $x$ system. This is not surprising due to the relatively poor scattering contrast between $\mathrm{K}$ and $\mathrm{Na}$ as well as the inherent disorder associated with any local ordering of this type (particularly as Na can adopt $a^{0} b^{0} c^{0}, a^{0} b^{+} c^{0}$ and $a^{-} b^{+} c^{-}$tilt states without significant energy penalty). Indeed, the appearance of continuous rods of diffuse in the EDPs from this structure (see Figs. 4-6) suggest almost perfect disorder, though quantitative measurement of the distribution of intensity along the 1-D rods of diffuse intensity is very difficult.

The essentially continuous nature of the changes in EDPs with composition (see e.g., Fig. 5) do not suggest any kind of morphotropic phase boundary enhanced behaviour (through polarisation rotation or fine scale twinning) in this $\mathrm{KNN} x$ system. Note that although $x=0.4$ is listed as the point of disappearance of the $\boldsymbol{b}^{+}$ tilting in Baker et al. [12], we still observe a weak, but sharp, spot there up to $x=0.46$ (see Fig. 5(a)). The 'exact' point of appearance or disappearance of the $\mathbf{G} \pm\left[{ }^{1} / 2,0,{ }^{1} / 2\right]_{p}{ }^{*}$ and $\mathbf{G} \pm\left\langle{ }^{1} / 2,1 / 2,1 / 2\right\rangle{ }_{p}{ }^{*}$ satellite reflections is clearly extremely difficult to define, due to the disordered diffuse scattering through these points of reciprocal space (see Fig. 5). The clear appearance of long range ordered $\mathbf{G} \pm\langle 1 / 2,1 / 2,1 / 2\rangle_{p}{ }^{*}$ satellite reflections at $x<\sim 0.19$ is, however, quite abrupt (cf. e.g., Fig. 5(c) with Fig. 5(d)). During synthesis it was noted that the $x=0.19$ composition densified far more readily than the $x=0.21$ and 0.17 samples suggesting a 'phase transition' at this composition. The expected ferroelectric polarisation direction at this boundary region, however, does not alter. This implies that any enhancement of properties will not be as significant as in a substance such like $\mathrm{Pb}_{1-\chi} \mathrm{Zr}_{x} \mathrm{TiO}_{3}$. This assessment of behaviour is in agreement with a recent work by Pang et al. [28], which showed that there was no significant change in properties from $x=0.4$ to 0.5 .

\section{References}

[1] Y. Saito, H. Takao, T. Tani, T. Nonoyama, K. Takatori, T. Homma, T. Nagaya, M. Nakamura, Nature 432 (2004) 84-87.

[2] H.E. Mgbemere, R.P. Herber, G.A. Schneider, J. Eur. Ceram. Soc. 29 (2009) 3273-3278.

[3] A.S. Kandari, A. Bhandari, A.A. Bourai, N.S. Panwar, Ferroelectrics 386 (2009) 139-151.

[4] S. Zhang, R. Xia, T.R. Shrout, G. Zang, J. Wang, Solid State Commun. 141 (2007) 675-679.

[5] A.W. Hewat, J. Phys. C 6 (1973) 2559-2572.

[6] R. Comes, M. Lambert, A. Guinier, Acta Crystallogr., Sect. A 26 (1970) 244-254.

[7] R. Comes, M. Lambert, A. Guinier, Solid State Commun. 6 (1968) 715-719.

[8] M. Lambert, R. Comes, Solid State Commun. 7 (1969) 305-308.

[9] S.R. Phillpot, M. Sepliarsky, M.G. Stachiotti, R.L. Migoni, S.K. Streiffer, J. Mater. Sci. 40 (2005) 3213-3217.

[10] H. Krakouer, R. Yu, C. Wang, K.M. Rabe, U.V. Waghmare, J. Phys. Condens Matter 11 (1999) 3779-3787.

[11] M. Ahtee, A.M. Glazer, Acta Crystallogr., Sect. A 32 (1976) 434-446.

[12] D.W. Baker, P.A. Thomas, N. Zhang, A.M. Glazer, Appl. Phys. Lett. 95 (2009) 091903-3.

[13] F. Denoyer, R. Comes, M. Lambert, Acta Crystallogr., Sect. A 27 (1971) 414-420.

[14] Z. Yi, Y. Liu, M.A. Carpenter, J. Schiemer, R.L. Withers, Dalton Trans. 40 (2011) 5066-5072.

[15] Sietronics Pty Ltd., Belconnen, ACT, Australia.

[16] P.M. Woodward, J. Appl. Crystallogr. 30 (1997) 206-207.

[17] D.W. Baker, P.A. Thomas, N. Zhang, A.M. Glazer, Acta Crystallogr., Sect. B 65 (2009) 22-28.

[18] N.E. Brese, M. O’Keeffe, Acta Crystallogr., Sect. B 47 (1991) 192-197.

[19] V.A. Shuvaeva, K. Yanagi, K. Yagi, K. Sakaue, H. Terauchi, Solid State Commun. 106 (1998) 335-339.

[20] D. Vanderbilt, M.H. Cohen, Phys. Rev. B 63 (2001) 094108

[21] M. Ahtee, A.W. Hewat, Acta Crystallogr., Sect. A 31 (1975) 846-850.

[22] R.L. Withers, Adv. Imag. Elect. Phys. 152 (2008) 303-337.

[23] A.M. Glazer, Acta Crystallogr., Sect. B 28 (1972) 3384-3392.

[24] N. Zhang, H. Yokota, A.M. Glazer, P.A. Thomas, Acta Crystallogr., Sect. B 67 (2011) 461-466.

[25] I.D. Brown, The Chemical Bond in Inorganic Chemistry: The Bond Valence Model, Oxford University Press, 2006.

[26] J.M. Perez-Mato, M. Aroyo, A. Garcia, P. Blaha, K. Schwarz, J. Schweifer K. Parlinski, Phys. Rev. B 70 (2004) 214111.

[27] I. Etxebarria, J.M. Perez-Mato, A. Garcia, P. Blaha, K. Schwarz, J. RodriguezCarvajal, Phys. Rev. B 72 (2005) 174108

[28] X. Pang, J. Qiu, K. Zhu, J. Am. Ceram. Soc. 94 (2011) 796-801. 